\title{
EL DESARROLLO DE LAS VENTAJAS COMPETITIVAS DE PORTER Y LOS IPAS VERDES EN EL COMERCIO EXTERIOR DEL PERÚ
}

Jorge Nicanor Arroyo Prado

\section{Resumen}

Se describe y amplía los atributos del Diamante de Porter para crear ventajas competitivas de las naciones con dos atributos más: "El desarrollo Sostenible" y "La Competitividad Verde", aplicable a los países en vias de desarrollo, y se analiza cada uno de ellos orientados al comercio exterior del Perú. Se propone una visión de bases para un comercio exterior competitivo así como el papel que debe desarrollar el Gobierno bajo esta visión.

Palabras clave: Desarrollo Sostenible, Competitividad Verde, Generación de Valor y Competitividad, Propuesta de Visión, Papel del Gobierno.

\section{Abstract}

This paper describes and extends the attributes of Diamond's Porter to create competitive advantages of nations, with two attributs more "Sustainable Development" and "Green Competitiveness" applicable to countries in process of development and analyzed each, oriented to foreign trade of Peru, also its proposes a vision for a competitive basis for foreign trade and the Government role should have in this vision proposed.

Key words: Sustainable Development, Green Competitiveness, Value Creation and Competitiveness proposed of Vision, the Government role. 


\section{EL POR QUÉ DE LA COMPETITIVIDAD}

En 1990, Michael E. Porter publicó "La Ventaja Competitiva de las Naciones", libro que tuvo gran impacto en los ambientes académicos, empresariales y gubernamentales de todo el mundo.

Buscando determinar cuáles son "los atributos de una nación que fomentan las ventajas competitivas en determinados sectores", el profesor Porter elaboró un modelo que permite comprender el proceso para lograr ventajas competitivas internacionales $\mathrm{y}$, a la vez, identificar la manera cómo los países pueden desarrollar su competitividad; es decir, mejorar la cantidad y calidad de los recursos que poseen y la capacidad para utilizar esos recursos de manera más eficiente. La crítica de sus opositores se centra en el hecho de que este libro tiene como base la investigación, evaluación y resultados con empresas corporativas de países desarrollados.

¿Será posible que los conceptos de "ventaja competitiva de las naciones" sean aplicables a un país en vías de desarrollo como el Perú?

La respuesta la daremos intentando adaptar estos conceptos en una visión en el que el Comercio Exterior Peruano, teniendo como objetivo fundamental el desarrollo sostenible y la democracia, así como su inserción a las normas ambientales internacionales y al comercio internacional, siempre que contribuya a lograr el bienestar general de los ciudadanos del Perú.

\section{EL MODELO DE PORTER AMPLIADO}

Según Michael Porter, la competitividad de un país y sus empresas puede definirse como la capacidad de diseñar, producir y comercializar bienes y servicios mejores y/o a buen precio que los de la competencia internacional.

Si se alcanza este logro, se reflejará en una evidente mejora del nivel de vida de la población. De acuerdo a esta definición, la idea básica se centra en que la competitividad no se hereda, ni depende de la coyuntura económica, sino hay que crearla con esfuerzo e iniciativa propia.

Consideramos que lo que hace próspero a un país es la capacidad de sus empresas para alcanzar elevados niveles de productividad; es decir, la capacidad de usar con eficiencia y creatividad la mano de obra, los recursos naturales y el capital, respetando el medio ambiente.

En el enfoque de Porter no importa tanto qué y cuántos recursos se poseen, sino qué se hace con los que se tienen. Esto conlleva a que en los países en vías de desarrollo como el Perú, las empresas que busquen ser competitivas no son aquellas que descubren un nuevo mercado o la tecnología más adecuada de producción, sino las que implementen o adapten los mejores cambios en la forma más rápida posible, como las normas técnicas y ambientales que los países desarrollados tienen para sus productos.

De acuerdo con Porter, el que una nación cuente con sectores capaces de 
competir exitosamente en el mercado internacional depende en gran medida del contexto que rodea a las empresas que conforman cada sector, pues estas no son entes aislados.

\section{EL DIAMANTE DE PORTER AMPLIADO}

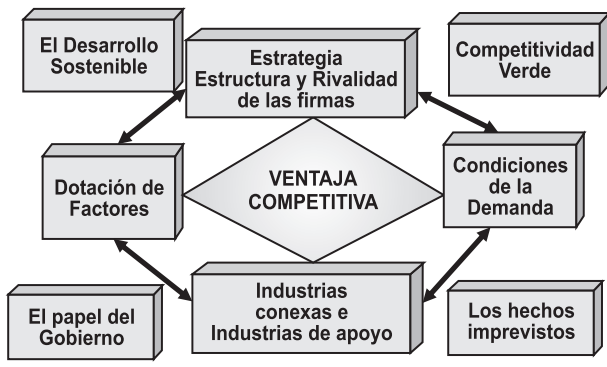

Gráfico $\mathbf{N}^{\circ} 1$

El Diamante de Porter Ampliado

El Diamante de Porter, eje de sus conceptos, se sustenta en que las empresas de un país siempre estarán determinadas por la interacción de cuatro grupos de atributos invariables:

1. La dotación de factores;

2. Las condiciones de la demanda;

3. Los proveedores, las industrias relacionadas y de apoyo; y

4. Las estrategias, estructuras y rivalidad de las empresas.

Para completar su Diamante, en el entorno en que se ubican estas empresas, Porter, reconoce la importancia y existencia de dos elementos adicionales:

5. Los hechos imprevistos.

6. El papel del Gobierno.

Sin embargo, para el caso de las empresas del país, sin modificar el Diamante de Porter, se puede complementar el entorno con dos elementos adicionales que hagan viable su aplicación a realidades empresariales como la peruana:

7. El Desarrollo Sostenible.

8. La Competitividad Verde.

En este modelo, la base de la competitividad o la capacidad para competir no deriva de los cuatro atributos, ni siquiera del conjunto de atributos considerados como un todo, sino de su interrelación, de su reforzamiento mutuo. Esto significa que el efecto que cada uno de ellos pueda causar depende del estado de los otros, que las ventajas de uno puedan crear o perfeccionar ventajas en otros, y que las desventajas de uno puedan acarrear desventajas en los otros. A este concepto de total dinamismo, Porter lo denominó el "Diamante de la Competitividad".

Los sectores exitosos en la economía internacional según Porter son aquellos a los cuales el Diamante de la Competitividad les es favorable. Sin embargo, esta afirmación no implica que todas las empresas sean exitosas; más aún, cuanto más competitivo sea el entorno, tanto más probable será que algunas de estas se queden en el camino, ya que no todas tienen iguales habilidades ni explotan similarmente el entorno.

\subsection{La dotación de factores}

El primer atributo del Diamante de Competitividad, se refiere a la situación del país respecto de lo que comúnmente se llama "dotación de los factores" (recursos humanos, recursos físicos, conocimientos, capital e infraestructura); es decir, a los in- 
puts necesarios para competir en una industria. Las condiciones de los factores no dependen solo de los factores en sí, sino del grado de eficiencia y efectividad con que se les armonice hacia el objetivo de producir un bien altamente competitivo.

Por otro lado, no todos los factores tienen la misma importancia para la obtención de la competitividad. Para explicar esta idea, Porter clasifica los factores en básicos y avanzados, por un lado; y en generalizados y especializados, por otro.

Los factores básicos comprenden los recursos naturales, el clima, la situación geográfica, la mano de obra no especializada o poco especializada. Los factores avanzados comprenden la infraestructura, los recursos humanos altamente especializados y el soporte en ciencia y tecnología. Los generalizados son los que pueden aplicarse a varias actividades, mientras los especializados tienen un radio de acción circunscrito, como es el caso de las empresas exportadoras que deben ser calificadas por tener una "producción más limpia” ambientalmente, también llamados productos verdes.

Así tenemos que el objetivo de una visión de una empresa agroexportadora, como en el caso de la agricultura en el Perú, tiene como factores básicos a considerar: las variadas condiciones climatológicas (microclimas) y la disponibilidad de tierras, así como el uso y disponibilidad de mano de obra no capacitada. Los factores avanzados serían los proyectos de irrigación y la infraestructura creada para explotar las tierras. La investigación tecnológica en un campo específico, como el organismo genéticamente modificado (OGM), usado en el Centro Internacional de la Papa o del Instituto del Espárrago, constituyen factores especializados.

En tanto que los factores básicos se heredan o se crean mediante inversiones modestas, los avanzados requieren grandes inversiones y esfuerzos de largo alcance.

En los últimos años, la globalización ha hecho menos esencial la disponibilidad de los factores básicos, pues hay gran movilidad de recursos y las empresas pueden trasladar sus unidades productivas ahí donde estos se encuentren. Hoy, la ubicación geográfica de las plantas industriales no se da solo en función de la localización de las materias primas, sino del lugar donde estas pueden agregar mayor valor al proceso productivo y también puedan proteger el medio ambiente.

A diferencia del Perú, pródiga en recursos naturales, los países asiáticos se vieron obligados a generar competitividad para poder acceder al mercado internacional, y lo lograron. Hoy, el país se enfrenta al reto de cómo evolucionar del viejo modelo rentista basado en recursos naturales y mano de obra barata, al desarrollo de ventajas competitivas que le permitan una adecuada inserción en la nueva economía mundial.

La apertura comercial ofrece muchas oportunidades para beneficiar al medio ambiente, principalmente mediante acceso a tecnologías limpias, 
mercados para productos verdes de exportación con mayor valor agregado y, generalmente, la integración más estrecha con un mundo preocupado con el tema ambiental;

La mayor parte de los impactos ambientales que comúnmente se relacionan con el medio ambiente están relacionados con el crecimiento económico, el que de todas maneras quisiéramos tener con o sin acuerdos comerciales, y para el cual necesitamos desarrollar nuestras normas y regulaciones ambientales de todas formas. Si bien es cierto que algo se está haciendo, es necesario actuar con mayor fuerza.

El efecto tecnológico, es muy probable que favorezca al medio ambiente. La presión competitiva internacional, la globalización de las prácticas empresariales y la inversión extranjera en general, fomentan un cambio productivo hacia tecnologias y gestiones más modernas y con menores impactos ambientales. La inversión extranjera muchas veces estimula la producción limpia en el país en desarrollo donde llega.

De estos argumentos no se debe concluir que no se requieren normas o regulaciones ambientales. Todo lo contrario. Si queremos crecer necesitamos estas normas y regulaciones, de todas maneras. La pregunta es: ¿Por qué no utilizar estratégicamente la coyuntura política que está ofreciendo paquetes para enfrentar la desaceleración, reactivar la economía y la negociación comercial, así como el Plan Nacional de Diversificación Productiva, el cual actualmente está presentando en consulta el Ministerio de la Producción? Esperamos que su resultado sea para proponer politicas integrales de sostenibilidad y que den inicio al Plan de Desarrollo a Largo Plazo.

Hay varios riesgos en esta coyuntura. Primero, se corre el peligro de distorsionar nuestras prioridades ambientales. Las iniciativas de política podrian supeditarse a los temas que preocupan a los grupos de interés en el extranjero, incluyendo los grupos proteccionistas, cuyas prioridades pueden ser diferentes a las nuestras. Segundo, al entrar en este juego se estaría reconociendo tácitamente que el medio ambiente y el comercio internacional están intimamente relacionados, asunto que hace más dificil resistir las presiones por incluir condiciones o salvaguardas "ambientales", no obstante que los Tratados Internacionales (TLC) proveen un entorno estable de las reglas de juego y sin barreras para el comercio y la inversión.

\subsection{Las condiciones de la demanda}

Por condiciones de la demanda se entiende la composición, el tamaño, ritmo de crecimiento y grado de sofisticación de las empresas en el mercado nacional, porque al estimularlas a la mejora y a la innovación, estas se convierten en determinantes de la competitividad.

Respecto de la composición, la idea es que un mercado interno segmentado, de compradores exigentes y de necesidades precursoras, puede presionar a las empresas al constante 
perfeccionamiento, como es tener una producción limpia. En cuanto al tamaño y al ritmo de crecimiento del mercado, las economías de escala que justifican la inversión en investigación y desarrollo pueden impulsar la competitividad al incentivar las inversiones.

Sin embargo, Porter también señala que un mercado interno pequeño puede convertirse en un incentivo para esforzarse por competir en el mercado externo. Por último, la sofisticación de los compradores o su internacionalización, se refiere a todo aquello que identifique y transmita sus gustos o hábitos de consumo en los productos que adquiere y que se les puede ofrecer.

En el caso peruano, dado el escaso poder adquisitivo y sin mayores exigencias del consumidor local, la demanda para muchos productos viene dada por el mercado externo, como sucede por ejemplo con los espárragos y el palmito, los que se exportan a diversos mercados internacionales, cumpliendo las normas ambientales. Ahora existen otros productos agroindustriales, como los cítricos, que se estan exportando después de muchos esfuerzos, aunque en la actualidad ha bajado la demanda.

\subsection{Los proveedores, las industrias relacionadas y de apoyo}

En el modelo de Porter, la competitividad de un sector se sustenta en la disponibilidad de los proveedores o industrias conexas que, a su vez, sean por sí mismos competitivos. Las industrias relacionadas comprenden a todas aquellas que comparten tecnologías comunes, insumos y productos complementarios; es decir, las industrias conexas son aquellas con las que las empresas pueden compartir o coordinar actividades de su cadena de valor, como el desarrollo de tecnología, fabricación, distribución, comercialización o servicio de producto.

Normalmente, en los países en desarrollo, dada la falta de proveedores competitivos, las industrias aparecen como sectores aislados en vez de clusters o industrias relacionadas.

Esto obliga a que la mayoría de los componentes tengan que importarse. En el Perú, significan alrededor del $50 \%$ de nuestras importaciones, por lo cual, las empresas se ven forzadas a procesos de integración vertical. Algunas veces, cuando no es posible obtener los insumos requeridos localmente, las industrias se ven obligadas a dedicarse a actividades que no forman parte de su negocio principal, como es el caso de la generación de electricidad o la operación de transporte en el sector minero formal peruano.

En el Perú, se tiene la presencia en el mercado de gran número de pequeñas y microempresas, caracterizadas por su flexibilidad. El reto es cómo integrar a estas empresas en el circuito de la cadena productiva para constituir cadenas o clusters de empresas a fin de lograr competitividad. El punto de partida es generar confianza, ya que vivimos en un país de desconfianza. Para ello, es necesario cambios estructurales profundos para sostener el crecimiento, de manera que no depen- 
dan solo de los gobernantes de turno sino que sean programas de largo plazo que respeten los sucesores, a fin de asegurar las reformas que logran la confianza.

\subsection{Las estrategias, estructuras y ri- validad de las empresas}

El último determinante de Porter de la ventaja competitiva de un país, es el contexto en el que se crean, organizan y gestionan sus empresas exportadoras, así como la naturaleza de la rivalidad entre ellas.

Parte de este contexto deriva del hecho de que en el ámbito nacional existen prácticas y enfoques de gestión comunes a las empresas. Porter menciona, entre otras, la formación y orientación de los líderes, el peso de la iniciativa individual, los medios para la toma de decisiones, la actitud hacia las actividades internacionales y la relación entre los trabajadores y los directivos.

De la misma manera, la idiosincrasia de una nación influye sobre las formas de organizar y gestionar las empresas y de alguna manera condiciona sus posibilidades de lograr ventajas competitivas. Los objetivos de las empresas y de las personas juegan también un papel importante, como pueden ser las motivaciones y los compromisos.

En cuanto a la rivalidad interna o doméstica de las empresas, Porter señala que la creación y persistencia de la ventaja competitiva en un sector determinado está asociada a una intensa rivalidad doméstica. La com- petencia en el mercado de origen sirve de estímulo a las empresas para que mejoren la calidad de sus productos o servicios, reduzcan precios e innoven.

En el caso peruano, el cambio hacia un entorno más competitivo se inicia en 1990, con la apertura y la liberalización de los mercados, la eliminación de las barreras de entrada y salida a los productos, así como la asunción de un rol menos protagónico del Estado. Estas fueron las condiciones necesarias para crear un mercado competitivo, además, de todo un trabajo complementario que evitara las prácticas anticompetitivas y los abusos frente a los consumidores.

Sin duda, el cambio hacia una cultura de mercado y el hecho de que las diferentes empresas exportadoras internalicen las nuevas reglas de juego, entre ellas las ambientales, no es tarea fácil. Algunos sostienen que es más fácil abrir los mercados que abrir las mentes, y que incentivar nuevas actividades y actitudes es verdaderamente una tarea compleja, que precisa de un cambio generacional y de una reforma de la educación desde su etapa inicial, lo cual conlleva tiempo. Pero esto es imprescindible.

\subsection{Los hechos imprevistos}

Los hechos imprevistos son factores adicionales que complementan el Diamante de la Competitividad. Precisamente, este fue uno de los hallazgos del estudio empírico de Porter.

Los acontecimientos imprevistos también ejercen influencia sobre la ventaja competitiva. Se dice que son 
los incidentes ajenos a una nación y sobre los cuáles las empresas o el gobierno no tienen mayor control.

La importancia de los hechos imprevistos para las ventajas competitivas radica en el hecho de que pueden alterar la posición competitiva de las naciones. Sin embargo, el que un hecho imprevisto se convierta en ventaja competitiva depende del diamante nacional. Porter hace ver que inclusive lo que puede parecer fruto de lo imprevisto es, en realidad, resultado de las diferencias de los entornos nacionales.

Entre estos hechos, Porter menciona los inventos, el talento creativo o recreativo de las personas, las discontinuidades tecnológicas, las discontinuidades en los costos de los insumos, los cambios significativos en los mercados financieros mundiales, o en el tipo de cambio, los aumentos insospechados de la demanda mundial o regional, las decisiones politicas de los gobiernos extranjeros y los conflictos bélicos, las convulsiones sociales o las acciones de terrorismo, como por ejemplo la crisis de los Estados Unidos de Norteamérica en 1998 y siguientes años, que ha afectado no solo a países en vias de desarrollo sino también a los países desarrollados, la crisis generalizada que ha frenado nuestro crecimiento notablemente por reducción de la demanda exterior: en abril del presente año hemos crecido solo el $2 \%$.

Otros hechos imprevistos que repercuten sobre el diamante nacional pueden ser también los desastres naturales. Un caso relativamente recien- te fue el Fenómeno de El Niño, que afectó el entorno del Perú en los años 1997 y 1998.

\subsection{E1 papel del gobierno}

El otro factor adicional que completa el modelo de Porter, y al que se le otorga gran importancia, es el poder que tiene el Gobierno de un país de influir sobre el diamante a través de leyes, normas y políticas, lo cual afecta a cada uno de los determinantes de la competitividad. En la medida que el gobierno establece las reglas de juego, puede mejorar o deteriorar la ventaja nacional o alterar el clima nacional para la competitividad.

Porter sostiene que, si bien es tentadora la idea de colocar al gobierno como el sexto determinante del diamante, en realidad el papel del gobierno en la competencia internacional es el de influir -positiva o negativamentesobre los cuatro determinantes. Más aún, Porter advierte sobre el peligro de perder de vista que la influencia del gobierno sobre la ventaja competitiva es inevitablemente parcial, puede aumentar o disminuir las probabilidades de conseguir ventaja competitiva, pero no puede crearla; porque es tarea exclusiva de las empresas exportadoras.

Desde la perspectiva del modelo porteriano, se acepta que el papel del gobierno es proporcionar el marco propicio para un entorno favorable; es decir, actuar como agente promotor y responsable de dotar al país de infraestructura básica, desarrollo tecnológico y servicios básicos de educación y salud, para permitir a las empresas 
competir con ventaja en los mercados internacionales.

Consideramos que un aspecto que se debe tener en cuenta y es de gran importancia en el actual contexto latinoamericano, es que no basta con declarar la economía de mercado para que esta funcione. Hace falta también el adecuado funcionamiento de las instituciones gubernamentales, con reglas de juego definidas y transparentes, un Estado eficaz sin corrupción.

Una herramienta básica en los negocios internacionales para determinar cuán riesgoso o atractivo sea un país como destino de inversiones, es el denominado "riesgo-país". El efecto del aumento del riesgo-país es la exigencia de un mayor retorno o tasa de descuento para los proyectos de inversión. Ello significa que si el riesgo-país es alto, entonces muchas inversiones dejarán de ejecutarse o se llevarán a cabo solo aquellas que permitan una rápida recuperación del capital, lo que debe tener presente nuestro gobierno.

Un elemento importante del riesgopaís es el riesgo político institucional. Para el inversionista, es fundamental saber si en el país se aplican las leyes, si las decisiones judiciales son independientes e imparciales y si la administración de justicia es confiable. En suma, si se garantiza y respeta el marco institucional del país.

$\mathrm{El}$ adecuado funcionamiento de las instituciones genera un entorno jurídico confiable para las inversiones. Sin un óptimo sistema proveedor de seguridad jurídica para todos, el mercado no podría funcionar eficientemente.

\subsection{El desarrollo sostenible}

Para los países en vías de desarrollo, es necesario lograr que sus empresas alcancen ventajas competitivas, dentro de los conceptos que plantea Porter. Se tiene que ver cómo las decisiones politicas de los gobiernos han incorporado actualmente la idea precursora de lograr un desarrollo sostenible y, dentro de ella, que las empresas exportadoras contribuyan a lograr el bienestar nacional.

Se supone que este enfoque parte de la existencia de una conciencia pública que ha integrado en sus decisiones los aspectos económicos, sociales y ambientales; donde la preocupación ambiental debe estar arraigada en la sociedad, y prevalezcan los criterios de solidaridad social y equidad generacional.

El desarrollo se debe plantear basado en la consolidación de la democracia con formas de participación e inclusión ciudadana. De ser así, la gobernabilidad evolucionaría hasta lograr un sistema sostenible en el cual se tenga control sobre las decisiones socioeconómicas y ambientales.

El mercado seguiría siendo el principal mecanismo de asignación de los recursos, pero los precios incorporarían los costos sociales y ambientales. Las políticas de desarrollo se diseñarían sobre la base de una gama más amplia de herramientas económicas, y estarían basadas en la producción de bienes y servicios con valor agregado. Asimismo, se estrecharian con mayor dinamismo los vínculos comerciales con otros países desarrollados. Actualmente, la exportación de pro- 
ductos tradicionales en el Perú alcanza el $76 \%$ de nuestras exportaciones, en tanto que los no tradicionales, que se manufacturan y proporcionan mayor valor agregado, representan solo el $24 \%$, porcentajes que es altamente necesario revertir.

Paralelamente, se desarrollaría una cultura de prevención del deterioro ambiental y se produciría un cambio en los patrones de consumo, ya que el consumidor sería mas exigente y preferiría bienes y servicios producidos sobre la base de procesos productivos ambientalmente sostenibles. De esta manera, mejoraría el ingreso de los productores y la calidad de vida de la población. Igualmente, la innovación tecnológica respondería a la nueva demanda por tecnologias eficientes y limpias.

Además, se promovería el desarrollo de tecnologías propias sobre la base de la adaptación de las extranjeras. También se produciria una revalorización del conocimiento tradicional. De esta manera, las nuevas tecnologías y el aprovechamiento de los conocimientos tradicionales se orientarian a solucionar los problemas de pobreza y deterioro ambiental, lo que permitiría desarrollar nuevas actividades económicas sostenibles.

El gobierno emitiría normas que buscarian la eficiencia con la mayor equidad posible, lo que contribuiría a mejorar la gobernabilidad del país. Las relaciones entre el Estado, el sector privado y la sociedad civil, se llevarían a cabo de una forma transparente, lo que mejoraría los mecanismos de control y desarrollo de la sociedad.

\subsection{La competitividad verde}

$\mathrm{Si}$ las empresas exportadoras del país asumen que el desarrollo sostenible es un objetivo común entre todos los países del planeta, los llamados países desarrollados, tienen diseñadas y en aplicación políticas y normas de protección ambiental para sus empresas, ligadas al desarrollo sostenible de su nación, lo cual les ha generado una ventaja competitiva en relación a los países en desarrollo. Este tema, por ser estrictamente ambiental, lo hemos denominado "Competitividad verde".

Aunque se podría argumentar que la competitividad verde podría ser también un componente de los factores avanzados del Diamante de Porter, consideramos que debemos aceptar el hecho de que las empresas de los países que ya cuentan con infraestructura adecuada para manufacturar productos mediante una producción "limpia", cuyo proceso se basa en normas y estándares ambientales establecidos, se encuentran en ventaja competitiva con las empresas de las naciones en donde aún no han adoptado estas políticas y normas ambientales. Por esta razón, actualmente, las autoridades de los países desarrollados, al verse frente a una competencia de productos que ingresan a su país y que no cumplen con las calidades y condiciones de producción ambiental, en defensa de sus consumidores y productores, exigen que se cumplan con ellas.

Las empresas extranjeras de países desarrollados que se instalan en un país como en el Perú, llegan con exi- 
gencias ambientales propias, las cuales son más avanzadas que las previstas en la legislación del país. Muchas veces, la transferencia tecnológica y por el efecto demostración, se logra una producción "más limpia", por lo cual su trascendencia en el plano de la gestión ambiental nacional pueden tener un efecto positivo. Un ejemplo en este sentido es el sector minero formal, donde las compañias mineras extranjeras aplican medidas y normas ambientales más exigentes que las cumplidas por el empresario minero nacional, conforme lo exige la autoridad ambiental del sector minero.

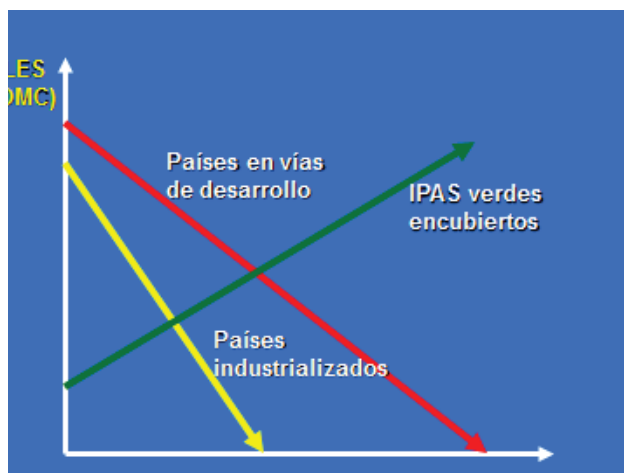

Figura $\mathrm{N}^{\circ}$ 2. La Liberalización del Comercio y los IPAS Verdes encubiertos

Sin embargo, para el caso de las empresas exportadoras de otros sectores productivos, surge la necesidad de diseñar y elaborar sus productos siguiendo las normas ambientales de los países que son mercados a fin de tener una aceptación que facilite el negocio. El no adaptar dichas normas ambientales hace que en las negociaciones comerciales con el país receptor este exija su cumplimiento para ingresar a su mercado. Este hecho, si no se atiende previsoramente, no solo es una ventaja competitiva respecto a nuestro país, sino que además se transformará en encubiertos instrumentos para arancelarios IPAS, a los que hemos denominado "verdes" por su relación con el medio ambiente. Según la Organización Mundial del Comercio (OMC), los aranceles de sus 159 países miembros deberian ser cero en el año 2020, mientras que los países industrializados debieron tener aranceles cero en el año 2010, lo que no se ha cumplido.

\section{UNA VISIÓN DE BASES PARA UN COMERCIO EXTERIOR COMPETITIVO E INCLUSIVO}

El considerar cada tema tratado, siguiendo los atributos ampliados del Diamante de la Competitividad de Michael Porter, nos permite proponer la síntesis de una visión de lo que queremos ser y cómo lo vamos hacer, para lograr un comercio exterior exitoso. Esto es importante a todo nivel y, en especial, para las empresas exportadoras, porque si no contamos con una Visión Nacional, no hay forma de saber adónde nos estamos dirigiendo.

La visión es un término que resulta de gran importancia en la elaboración de una estrategia nacional. Una visión se plasma en un conjunto de planes o acciones que llevan a una decisión óptima en un país. Los planes estratégicos dan pautas concretas que permiten hacer realidad la "visión" planteada para el país. Asimismo, es importante resal- 
tar que la visión debe ser entendida y compartida por todos los ciudadanos

De nada sirve que solo burócratas calificados de "alto nivel" expliquen su visión si los actores sociales, como el sector público, el sector privado, el sector académico y, en especial, las empresas exportadoras, no la sienten como suya. La visión debe gestarse en la base de los sectores productivos $\mathrm{y}$, en general, de la percepción de los actores, a fin de cohesionarlos en su conjunto para la acción. La única manera de tener éxito y poder afrontar los retos del futuro es que los líderes empresariales y politicos hagan realidad una visión compartida con participación de toda la población, incluyendo el sector académico. Esto implica el compromiso de todos los agentes económicos y políticos para concentrar los esfuerzos en la misma dirección.

La visión debe reflejar los valores, creencias y conductas del país, siendo necesario cambiar algunos de ellos en la búsqueda de una nueva "cultura de generación de valor y competitividad". Las ventajas de contar con una visión de futuro compartida son muchas, entre las que destacan las siguientes:

- Situarse adecuadamente en el mundo, es decir, analizar y entender el contexto internacional en el cual estamos insertos, y definir cómo nos vamos a insertar mejor.

- Adoptar una perspectiva y una actitud de mediano y largo plazo, dejando de lado el excesivo sesgo en el análisis y en las propuestas cortoplacistas.
- Utilizar adecuadamente todos los recursos disponibles: recursos físicos, naturales, humanos, tecnológicos, económico-financieros, políticos, diplomáticos, militares y culturales, en el marco de un desarrollo sustentable, a fin de hacer realidad la visión planteada.

- Formar un sentido de identidad nacional y reforzar la auto-estima y responsabilidad, de modo que permita a la sociedad definirse en una dirección positiva.

El tener una visión nos da una referencia de la verdadera dimensión de la tarea del desarrollo. Tal vez lo más importante es que una visión compartida lleva al compromiso de todas las personas involucradas y a la toma de conciencia de que todos necesitan de todos.

De otro lado, la metodología del Diamante de Competitividad de Porter, como la hemos planteado, ayuda a gestar la visión compartida para cada actor que participa en el comercio exterior.

Según Porter, los mismos elementos que determinan las ventajas competitivas de una nación son válidos $\mathrm{y}$ aplicables al caso del comercio exterior y las empresas exportadoras, ya que, en estos casos, la ventaja competitiva surge de la interacción entre las condiciones nacionales y locales.

El nuevo tratamiento de generación de valor y de competitividad parte del cambio de las actitudes mentales de los líderes. Solo una población con una actitud competitiva de valor, en- 
tendiendo la nueva dinámica de la economía mundial en la que los recursos naturales y la mano de obra barata ya no son lo más importante para competir en el mundo, es la que va a permitir desarrollar el potencial del comercio exterior.

La forma de actuar de las personas obedece a su experiencia pasada, a sus creencias, al contexto en el cual ve "su mundo", aunque muchas veces este no responde a la realidad, por lo cual el éxito del ayer muchas veces se convierte en el fracaso de hoy. Por ejemplo, los empresarios peruanos trabajaron por muchos años en una economía cerrada, protegida e inestable, donde el Estado era el Estratega Empresarial. En este contexto, la información y los lobbies eran la clave del éxito de las empresas, y la estrategia era el bajo costo; entonces, era válido buscar la máxima rentabilidad en las relaciones con los clientes, los proveedores y los recursos. El resultado fue obtener rentabilidad, pero con poca innovación de los productos y visión del largo plazo.

Posteriormente, cuando se abrió la economía y se terminaron los subsidios y las tasas de cambio preferenciales, como los dólares muc, los resultados fueron la baja rentabilidad y pérdida del mercado interno. No estaban preparados para la innovación, la generación de valor y la competitividad, donde la clave del éxito era trabajar con una estrategia de alianzas con proveedores, distribuidores y clientes, es decir, compartiendo información. Los empresarios seguían trabajando con los esquemas del pasado, los cuales, en el nuevo contexto, ya no eran válidos. En consecuencia, el tránsito o cambio del "éxito" al "fracaso" fue un hecho evidente; es decir, el éxito del ayer se convirtió, en aquel momento, en el causante del fracaso de hoy.

Lo más evidente es que el pasado ya no sirve para predecir el futuro, pero lo más grave es que nos distrae o confunde sobre lo que es verdaderamente relevante hoy en día, en un mundo globalizado y de grandes cambios tecnológicos. Siendo la base de la creación de riqueza la economía de mercado, paradójicamente, en muchos casos, esta resulta siendo mal vista y no aceptada.

Hay variables culturales que llevan a una confrontación entre la riqueza y la pobreza. Tal vez el hecho de que, históricamente, la riqueza haya estado en muchos casos asociada a la explotación o la corrupción, lleva a no verla bien. En este contexto, el cambio profundo tiene que darse, y la riqueza obtenida con trabajo, esfuerzo, innovación, creación de valor y creatividad tiene que ser bienvenida, necesitando el país de creadores de riqueza bajo este principio, que es la base de la competitividad dentro del marco de un desarrollo sustentable.

Un esquema mental muy difundido es que no sólo estamos insatisfechos con lo que somos, sino que tampoco deseamos que los demás sean exitosos. El éxito resulta castigado y en muchas organizaciones es mal visto, y no se le permite, tanto al empresario como al líder, sobresalir o destacar. 
En conclusión, ¿cuál debe ser el papel del gobierno bajo esta visión?

Las tareas pendientes del Gobierno como agente facilitador y habilitador de condiciones favorables para la competitividad en el comercio exterior, son:

- Facilitar y propiciar el desarrollo de la denominada "cultura de creación de valores y de competitividad", a fin de que sean los mismos agentes económicos quienes tomen la decisión y construyan una economía competitiva en el marco del desarrollo sustentable y la protección ambiental.

- Proporcionar una visión positiva de lo que se debe hacer como pais, con un mensaje coherente y consistente hacia el futuro a largo plazo.

- Proporcionar reglas de juego claras, que permitan abrir un consenso sobre las líneas maestras de la economía del país sin proteccionismos orientados que perjudican la credibilidad y, por lo tanto, la confianza.

- Mejorar la calidad y la disponibilidad de la infraestructura de servicios básicos para lograr mayor productividad, con la intervención, acción decidida $\mathrm{y}$ transversal de todos los sectores gubernamentales, en el que cada uno cumpla su verdadero rol.

- Proporcionar una adecuada seguridad jurídica y mejorar el buen desempeño de las instituciones que faciliten el desarrollo de la competitividad, desterrando definitivamente la corrupción.

- Generar un ambiente que desarrolle la innovación y propicie una mayor competencia de la economía en general, mediante incentivos a la inversión para la generación de capital y la capacitación y perfeccionamiento a todo nivel. 


\section{REFERENCIAS BIBLIOGRÁFICAS}

Guisado, Tato Manuel (2000). Internacionalización de la Empresa. Madrid. Editorial Pirámide.

Hill, Charles W. L. (2011). Negocios Internacionales. Competencia en el Mercado Global. México. Mc Graw Hill.

Instituto Cuanto - USAID. (2000). El Medio Ambiente en el Perú-Año 2000. Perú.

Naisbitt, J. \& Alburdene, P. (1994). Megatendencias 2000. Bogotá. Editorial Norma.

Organización Mundial del Comercio (2000). El Comercio hacia el Futuro. Ginebra. 2da. Edición OMC.

Peng, Mike W. (2012). Negocios Globales. México. 2da. Edición. Editorial CENGAGE.

\section{PÁGINAS WEB:}

Sociedad Nacional de Industrias www.sni.org.pe Organización Mundial del Comercio www.wto.org Ministerio del Ambiente www.minam.gob.pe Ministerio de la Producción www.produce.gob.pe 